\title{
CORRECTIONS
}

\section{GPs are warned over unnecessary migraine referrals}

In this News article by Geoff Watts (BMJ 2011;342:d1798, doi:10.1136/bmj.d1798) we wrongly reported that at a recent seminar in London, Timothy Steiner, a headache specialist at Imperial College London, said that only one in 10 patients with migraine needed to be referred to a neurologist. If fact, the figure he actually cited was one in 100 .

Cite this as: $B M J$ 2011;342:d2315 